\title{
Origin of the prestellar core mass function and link to the IMF - Herschel first results
}

\author{
Ph. André ${ }^{1}$, A. Men'shchikov ${ }^{1}$, V. Könyves ${ }^{1}$, and D. Arzoumanian ${ }^{1}$ \\ ${ }^{1}$ Laboratoire AIM, CEA Saclay, IRFU/Service d'Astrophysique, \\ F-91191, Gif-sur-Yvette, France \\ email: pandre@cea.fr
}

\begin{abstract}
We briefly review ground-based (sub)millimeter dust continuum observations of the prestellar core mass function (CMF) and its connection to the stellar initial mass function (IMF). We also summarize the first results obtained on this topic from the Herschel Gould Belt survey, one of the largest key projects with the Herschel Space Observatory. Our early findings with Herschel confirm the existence of a close relationship between the CMF and the IMF. Furthermore, they suggest a scenario according to which the formation of prestellar cores occurs in two main steps: 1) complex networks of long, thin filaments form first, probably as a result of interstellar MHD turbulence; 2) the densest filaments then fragment and develop prestellar cores via gravitational instability.
\end{abstract}

Keywords. stars: formation - ISM: clouds - ISM: structure - submillimeter

\section{Prestellar core mass functions from ground-based observations}

Wide-field (sub)mm dust continuum mapping is a powerful tool to take a census of prestellar dense cores and young protostars within star-forming clouds. At the end of the 1990s, the advent of bolometer cameras such as MAMBO and SCUBA on groundbased (sub)millimeter radio-telescopes like the IRAM 30m and the JCMT led to the identification of numerous cold, compact condensations (see Fig. 1-left for examples) that do not obey the Larson (1981) self-similar scaling relations and are intermediate in their properties between diffuse CO clumps and infrared young stellar objects (cf. Motte et al. 1998, 2001 and André et al. 2000, Ward-Thompson et al. 2007 for reviews). These (sub)millimeter continuum condensations detected from the ground are $\sim 3$ orders of magnitude denser than typical CO clumps (e.g. Kramer et al. 1998) and feature large ( $\gg 50 \%$ ) mean column density contrasts over their parent background clouds, strongly suggesting they are self-gravitating. The latter is directly confirmed by line observations in a number of cases. When available, the virial masses of the condensations indeed agree within a factor of $\sim 2$ with the masses derived from their (sub)millimeter dust continuum emission (e.g. André et al. 2007). A small fraction of these condensations lie at the base of powerful jet-like outflows and correspond to Class 0 protostars. However, the majority of them are starless/jetless and appear to be the immediate prestellar progenitors of individual protostars or protostellar systems.

As first pointed out by Motte, André, Neri (1998) in the case of the $\rho$ Ophiuchi (L1688) cloud, the core mass function (CMF) found for these starless dust continuum condensations is very similar in shape to the stellar IMF. While the early determination of the CMF by Motte et al. (1998), based on only 57 prestellar condensations, was clearly limited by small-number statistics, similar results were consistently found by a number of independent groups in the past decade and the statistics improved somewhat over the years (e.g. Testi \& Sargent 1998; Johnstone et al. 2000, 2001; Motte et al. 2001; Stanke et al. 

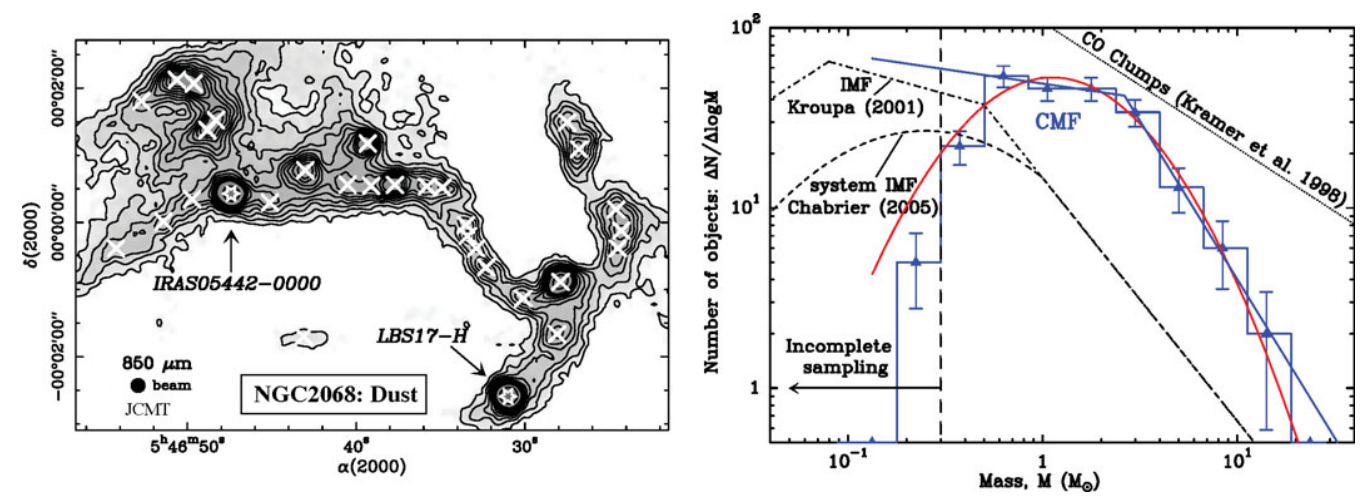

Figure 1. Left: SCUBA $850 \mu \mathrm{m}$ dust continuum map of the NGC 2068 protocluster extracted from the mosaic of Orion B by Motte et al. (2001). A total of 30 compact prestellar condensations (marked by crosses), with masses between $\sim 0.4 M_{\odot}$ and $\sim 4.5 M_{\odot}$, are detected in this $\sim 1 \mathrm{pc}$ $\times 0.7$ pc field. Right: Differential mass function [dN/dlogM] of the 229 starless dust continuum condensations detected at $850 \mu \mathrm{m}$ with SCUBA in the Orion A/B cloud complex excluding the crowded OMC1 and NGC 2024 regions (histogram with error bars - from Motte et al. 2001, Johnstone et al. 2001, and Nutter \& Ward-Thompson 2007). A two-segment power-law fit and a log-normal fit are shown for comparison. For reference, the field star IMF (Kroupa 2001), the IMF of multiple systems (Chabrier 2005), and the typical mass spectrum found for CO clumps (Kramer et al. 1998) are also shown.

2006; Nutter \& Ward-Thompson 2007; Enoch et al. 2008). There is thus good evidence in nearby star-forming regions such as Ophiuchus, Serpens, Orion A \& B, and Perseus that the shape of the prestellar CMF (e.g. Fig. 1-right) is consistent with the Salpeter power-law IMF at the high-mass end $\left(\mathrm{dN} / \mathrm{d} \log \mathrm{M} \propto \mathrm{M}^{-1.35}\right)$, and significantly steeper than the mass distribution of diffuse $\mathrm{CO}$ clumps $\left(\mathrm{dN} / \mathrm{d} \log \mathrm{M} \propto \mathrm{M}^{-0.7}-\right.$ e.g. Kramer et al. 1998). The difference presumably arises because CO clumps are primarily structured by supersonic turbulence (e.g. Elmegreen \& Falgarone 1996) while prestellar condensations are largely free of supersonic turbulence and clearly shaped by self-gravity (e.g. Motte et al. 2001, André et al. 2007). The slope of the observed CMF becomes shallower than the Salpeter power law and more similar to the slope of the CO clump mass distribution at the low-mass end. For instance, in the Orion A/B complex which contains the largest sample of starless submm continuum cores identified from the ground (229 objects), the entire prestellar CMF can be fit equally well with either a two-segment broken power law or a log-normal distribution down to the completeness limit of the observations (cf. Fig. 1-right - compare Nutter \& Ward-Thompson 2007 and André et al. 2009).

The median prestellar core mass observed in regions such as $\rho$ Ophiuchi and Orion $\left(\sim 0.2-1.5 M_{\odot}\right)$ is only slightly larger than the characteristic $\sim 0.5 M_{\odot}$ set by the peak of the IMF in $\mathrm{dN} / \mathrm{d} \log \mathrm{M}$ format (cf. Fig. 1-right). Such a close resemblance of the CMF to the IMF in both shape and mass scale is consistent with the view that the prestellar condensations identified in (sub)millimeter dust continuum surveys are about to form stars on a $\sim$ one-to-one basis, with a fixed and high local efficiency, i.e., $M_{\star}=\epsilon_{\text {core }} M_{\text {core }}$ with $\epsilon_{\text {core }} \sim 30-100 \%$. Taken at face value, this finding suggests that the IMF is at least partly determined by cloud fragmentation at the prestellar core stage (or earlier).

Interestingly, in their near-IR extinction imaging study of the Pipe dark cloud, Alves et al. (2007) found a population of 159 starless cores whose mass distribution similarly follows the shape of the IMF. This finding is reminiscent of the CMF results obtained with ground-based (sub)millimeter dust continuum observations, although most of the starless cores in the Pipe Nebula are gravitationally unbound objects confined by external 
pressure (Lada et al. 2008). Hence, a large fraction of them do not qualify as prestellar cores and may never evolve into stars. Assuming nevertheless that most of them will evolve into self-gravitating prestellar cores and form stars, the Alves et al. (2007) result suggests that the IMF may be determined even earlier than the prestellar core stage.

Appealing as a direct connection between the prestellar CMF and the IMF might be, several caveats should be kept in mind. First, although core mass estimates based on optically thin (sub)millimeter dust continuum emission are straightforward, they rely on uncertain assumptions about the dust (temperature and emissivity) properties (e.g. Stamatellos et al. 2007). Second, existing ground-based determinations of the CMF are limited by small-number statistics at both ends of the mass spectrum. In particular, this has led to concerns that the shape of the CMF may be strongly affected by incompleteness effects at the low-mass end (e.g. Johnstone et al. 2000). Third, there are hints that the star formation efficiency at the level of an individual core $\left(\epsilon_{\text {core }}\right)$, corresponding to a global shift in mass scale between the CMF and the IMF, may vary from cloud to cloud, with values ranging from $\epsilon_{\text {core }} \sim 15 \%$ in Taurus (Onishi et al. 2002), to $\epsilon_{\text {core }} \sim 30 \%$ in the Pipe (Alves et al. 2007), to $\epsilon_{\text {core }} \sim 50-100 \%$ in Ophiuchus (Motte et al. 1998). It is presently unclear whether this reflects real variations of $\epsilon_{\text {core }}$ with environment or whether this results from, e.g., the difficulty of adopting a uniform, precise definition of the boundaries between individual cores and the local background cloud when comparing different core samples.

With an angular resolution at 70-300 $\mu \mathrm{m}$ comparable to, or better than, the largest ground-based millimeter-wave radio-telescopes, the Her schel Space Observatory successfully launched by ESA in May 2009 (Pilbratt et al. 2010), now makes it possible to address the above issues on the CMF-IMF connection. In particular, Herschel can dramatically improve on the statistics of the CMF based on deep, extensive surveys of nearby clouds. Herschel can also help to greatly reduce the uncertainties in the core masses through direct measurements of the dust temperatures. In the following sections, we summarize and discuss the first results from the Gould Belt survey, one of the largest key projects with Herschel, whose main initial motivation is precisely to clarify the nature of the relationship between the CMF and the IMF (cf. André \& Saraceno 2005).

\section{First results from Herschel on the CMF-IMF connection}

The observational objective of the Herschel Gould Belt survey is to image the bulk of nearby $(d \lesssim 500$ pc) molecular clouds, mostly located in Gould's Belt (e.g. Guillout 2001), at 6 wavelengths between $70 \mu \mathrm{m}$ and $500 \mu \mathrm{m}$. The total surface area covered by the survey will exceed $160 \mathrm{deg}^{2}$ (cf. André et al. 2010 and references therein). Two extreme regions among a sample of 15 nearby cloud complexes were selected for imaging during the science demonstration phase of Herschel in October 2009: the Polaris Flare and the Aquila Rift. While the Polaris flare field is a high-latitude translucent cloud with little to no star formation at $d \sim 150$ pc (e.g., Heithausen et al. 2002), the Aquila field is a very active star-forming complex at $d \sim 260 \mathrm{pc}$ (e.g., Gutermuth et al. 2008). Our initial results for these two regions are discussed in 6 papers published in the A\&A special issue on Herschel (André et al. 2010, Könyves et al. 2010, Bontemps et al. 2010, Miville-Deschênes et al. 2010, Men'shchikov et al. 2010, Ward-Thompson et al. 2010).

Briefly, 302 starless cores but no protostars were detected with Herschel in the Polaris field $\left(\sim 8 \mathrm{deg}^{2}\right)$. The locations of the Polaris starless cores in a mass versus size diagram (Fig. 2-left) shows that they are $\sim 2$ orders of magnitude less dense than self-gravitating isothermal Bonnor-Ebert spheres and therefore cannot be gravitationally bound. The mass function of these unbound starless cores peaks at an order of magnitude smaller 

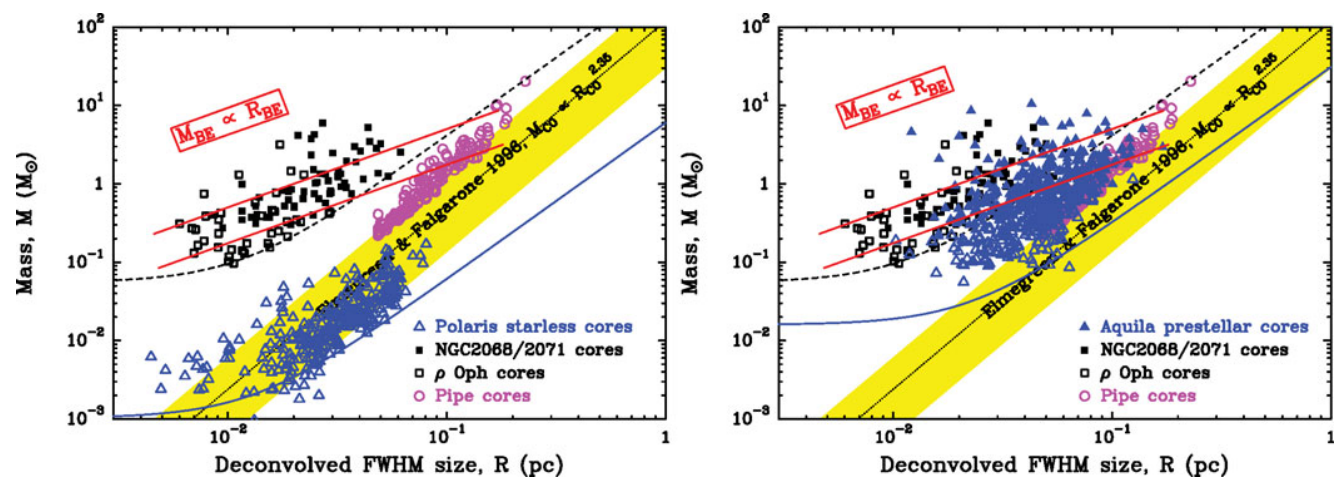

Figure 2. Mass vs. size diagrams for the starless cores detected with Herschel-SPIRE/PACS in Polaris (left) and Aquila (right) (triangles, from André et al. 2010 and Könyves et al. 2010). For comparison, the locations of the (sub)mm continuum prestellar cores identified by Motte et al. $(1998,2001)$ in $\rho$ Oph and NGC2068/2071, respectively, are shown, along with the dust extinction cores of the Pipe nebula (Alves et al. 2007, Lada et al. 2008), and the correlation observed for diffuse CO clumps (shaded band - cf. Elmegreen \& Falgarone 1996). The two solid lines $\left(M_{\mathrm{BE}} \propto R_{\mathrm{BE}}\right)$ mark the loci of critically self-gravitating isothermal Bonnor-Ebert spheres at $T=7 \mathrm{~K}$ and $T=20 \mathrm{~K}$, respectively. The 302 starless cores of Polaris lie much below the two Bonnor-Ebert lines in the left panel, suggesting they all are unbound. The 341 cores classified as prestellar by Könyves et al. (2010), out of a total of 541 starless cores in Aquila, are shown as filled triangles in the right panel. The $(5 \sigma)$ detection threshold at $d=150 \mathrm{pc}$ of existing ground-based (sub)mm (e.g., SCUBA) surveys as a function of size is shown by a dashed curve. The $5 \sigma$ detection thresholds of the SPIRE $250 \mu \mathrm{m}$ observations, given the estimated levels of cirrus noise and distances (150 pc for Polaris and $260 \mathrm{pc}$ for Aquila), are shown by solid curves.

mass than the stellar IMF (Fig. 3-left - André et al. 2010). In contrast, more than 200 (Class 0 \& Class I) protostars can be identified in the Herschel images of the whole $\left(\sim 11 \mathrm{deg}^{2}\right)$ Aquila field (Bontemps et al. 2010$)$, along with a total of 541 starless cores $(\sim 0.01-0.1 \mathrm{pc}$ in size). Most $(>60 \%)$ of these 541 starless cores lie close to the loci of critical Bonnor-Ebert spheres in a mass versus size diagram (Fig. 2-right), suggesting that they are self-gravitating and prestellar in nature (Könyves et al. 2010). The CMF derived for the entire sample of 541 starless cores in Aquila is well fit by a log-normal distribution and closely resembles the IMF (Fig. 3-right - Könyves et al. 2010, André et al. 2010). The similarity between the Aquila CMF and the Chabrier (2005) system IMF is consistent with a $\sim$ one-to-one correspondence between core mass and stellar system mass $\left(M_{\star \text { sys }}=\epsilon_{\text {core }} M_{\text {core }}\right)$. Comparing the peak of the CMF to the peak of the system IMF suggests that the efficiency $\epsilon_{\text {core }}$ of the conversion from core mass to stellar system mass is between $\sim 0.25$ and $\sim 0.4$ in Aquila, depending on whether one considers the reduced sample of the best 341 candidate prestellar cores or the entire sample of all 541 starless cores (see discussion in Könyves et al. 2010).

The early results of the Herschel Gould Belt survey therefore confirm the existence of a close relationship between the prestellar CMF and the stellar IMF, using data with already a factor of $\sim 2$ to 9 better counting statistics than the ground-based studies discussed in $\S 1$. These results seem difficult to reconcile with models in which competitive accretion plays a key role in shaping the distribution of stellar masses (e.g. Bate \& Bonnell 2005) and are in much better agreement with the gravo-turbulent fragmentation picture (e.g. Larson 1985, Klessen \& Burkert 2000, Padoan \& Nordlund 2002, Hennebelle \& Chabrier 2008). In any event, the observations clearly suggest that one of the keys to the problem of the origin of the IMF lies in a good understanding of the formation process of prestellar cores. This is true even if additional processes, such as rotational 

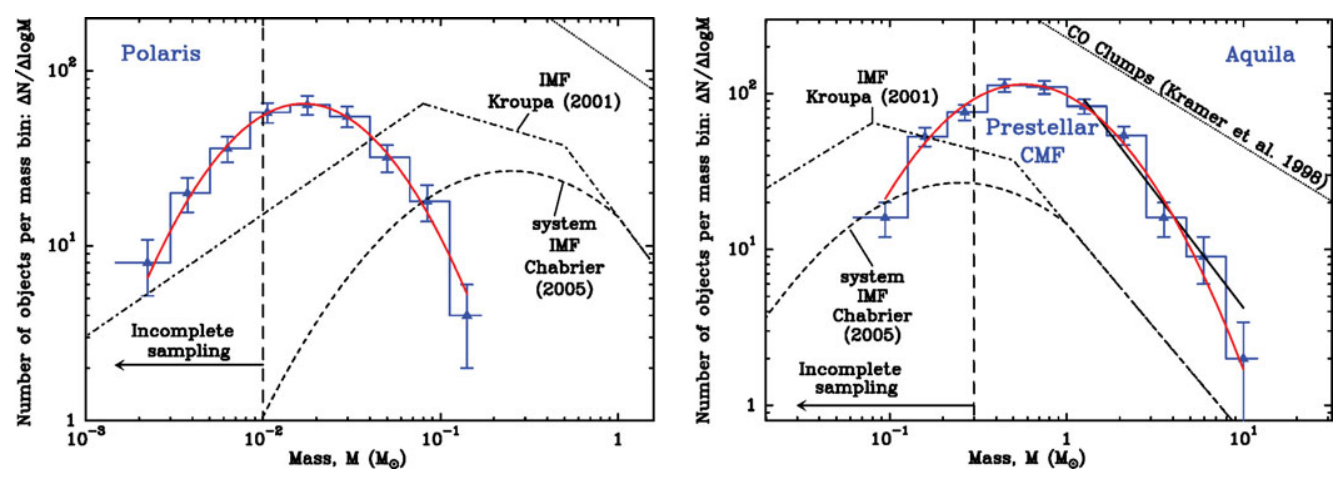

Figure 3. Core mass functions (histograms with error bars) derived from our Herschel observations of the Polaris (left) and Aquila (right) regions, which reveal of total of 302 starless cores and 541 candidate prestellar cores, respectively (André et al. 2010 and Könyves et al. 2010). The IMF of single stars (corrected for binaries - e.g., Kroupa 2001), the IMF of multiple systems (e.g., Chabrier 2005), and the typical mass spectrum of CO clumps (e.g., Kramer et al. 1998) are shown for comparison. Log-normal fits to the observed CMFs are superimposed. These fits peak at $\sim 0.02 M_{\odot}$ (Polaris) and $\sim 0.6 M_{\odot}$ (Aquila), and have standard deviations of $\sim 0.41$ and $\sim 0.43$ in $\log _{10} M$, respectively. (For reference, the log-normal part of the Chabrier system IMF peaks at $0.25 M_{\odot}$ and has a standard deviation of $\sim 0.55$ in $\log _{10} M$. .)

sub-fragmentation of prestellar cores into binary/multiple systems during collapse, probably also play an important role and may help to populate the low-mass end of the IMF (e.g. Bate et al. 2003, Goodwin et al. 2008).

\section{Spatial distribution of Her schel cores vs. large-scale cloud structure}

The high quality and dynamic range of the Herschel images are such that they provide key information on both dense cores on small $(<0.1 \mathrm{pc})$ scales and the structure of the parent background cloud on large $(>1 \mathrm{pc})$ scales. In particular, one of the most spectacular early findings made with Herschel is the fascinating omnipresence of long (> pc scale) filamentary structures in the cold interstellar medium and the apparently tight connection between the filaments and the formation process of dense cores (e.g. André et al. 2010, Men'shchikov et al. 2010, Molinari et al. 2010). In the Aquila Rift and Polaris flare clouds, for instance, an excellent correspondence is observed between the filaments and the spatial distribution of compact cores (see Men'shchikov et al. 2010 and Fig. 4). More precisely, the cores identified with Herschel are preferentially found within the densest filaments. In Aquila, the distribution of background cloud column densities for the prestellar cores shows a steep rise above $N_{\mathrm{H}_{2}}^{\text {back }} \sim 5 \times 10^{21} \mathrm{~cm}^{-2}$ (cf. Fig. 5-left) and is such that $\sim 90 \%$ of the candidate bound cores are found above a background column density $N_{\mathrm{H}_{2}}^{\text {back }} \sim 7 \times 10^{21} \mathrm{~cm}^{-2}$, corresponding to a background visual extinction $A_{V}^{\text {back }} \sim 7$. The Herschel observations of the Aquila Rift complex therefore strongly support the existence of a visual extinction threshold for the formation of prestellar cores (at $A_{V}^{\text {back }} \sim 5-10$ ), which had been suggested based on earlier ground-based studies of, e.g., Taurus and Ophiuchus (cf. Onishi et al. 1998, Johnstone et al. 2004). In the Polaris flare, our results are also consistent with such an extinction threshold since the observed background column densities are all below $A_{V}^{\text {back }} \sim 7$ and there are no examples of bound prestellar cores in this cloud. Another visualization of the extinction threshold is obtained by considering the probability density function (PDF) of column densities in the $\sim 11 \mathrm{deg}^{2}$ field observed with Herschel in Aquila (cf. Fig. 5-right). This column density PDF is reasonably well described by a log-normal distribution below $A_{V}^{\text {back }} \sim 7$ 

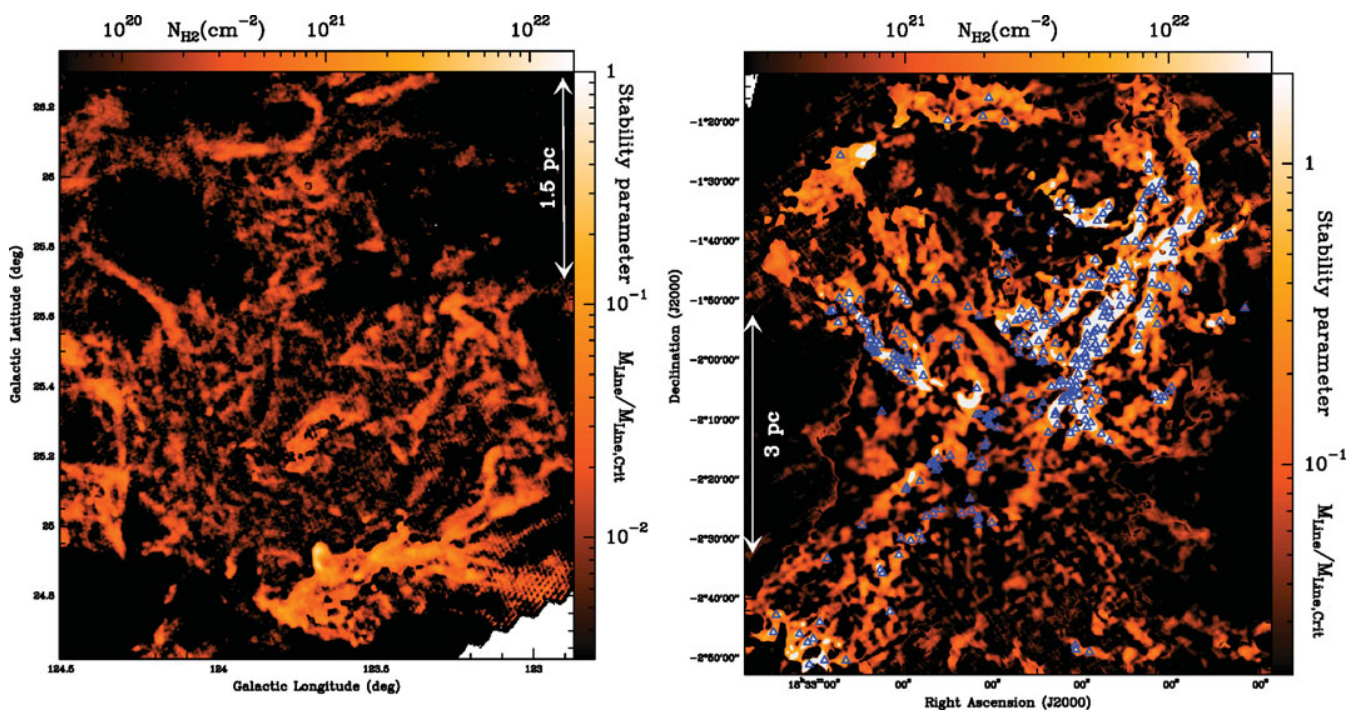

Figure 4. Column density maps of two subfields in Polaris (left) and Aquila (right) derived from our Herschel data (André et al. 2010). The contrast of the filaments with respect to the non-filamentary background has been enhanced using a curvelet transform (cf. Men'shchikov et al. 2010 and Starck et al. 2003). Given the typical width 10000-15000 AU of the filaments, these column density maps are equivalent to maps of the mass per unit length along the filaments. The scale shown on the right of each panel is given in approximate units of the critical line mass $2 c_{s}^{2} / G$ (cf. Inutsuka \& Miyama 1997). The areas where the filaments have a mass per unit length larger than half the critical value and are thus likely gravitationally unstable have been highlighted in white. The bound prestellar cores identified by Könyves et al. (2010) in Aquila are shown as small triangles in the right panel; there are no bound cores in Polaris.

and shows a pronounced power-law tail above $A_{V}^{\text {back }} \sim 7$. While similar column density PDFs have been recently reported from near-IR extinction studies (e.g. Kainulainen et al. 2009), the power-law tail at high column densities is particularly clear in the Herschel data of Aquila. Based on Fig. 5, we believe that this power-law tail reflects the dominant role of gravity above $A_{V}^{\text {back }} \sim 7$ and is intimately related to the formation of prestellar cores (see also $\S 4$ below).

\section{Implications for our understanding of the core formation process}

Our Herschel results in Polaris and Aquila also provide key insight into the core formation issue. They support an emerging picture (see, e.g., Myers 2009) according to which complex networks of long, thin filaments form first within molecular clouds, probably as a result of interstellar MHD turbulence (e.g. Padoan et al. 2001), and then the densest filaments fragment into a number of prestellar cores via gravitational instability. That the formation of filaments in the diffuse ISM represents the first step toward core/star formation is suggested by the filaments already being omnipresent in a diffuse, non-star-forming cloud such as Polaris (cf. Fig. 4-left, Men'shchikov et al. 2010, and Miville-Deschênes et al. 2010). The second step appears to be the gravitational fragmentation of a subset of the filaments into self-gravitating cores. Indeed, most $(>60 \%)$ of the bound prestellar cores and Class 0 protostars identified in Aquila are concentrated in gravitationally unstable filaments for which the mass per unit length exceeds the critical mass per unit length required for hydrostatic equilibrium (cf. Ostriker 1964, Inutsuka \& Miyama 1997), $M_{\text {line,crit }}=2 c_{s}^{2} / G \sim 15 M_{\odot} / \mathrm{pc}$, where $c_{s} \sim 0.2 \mathrm{~km} / \mathrm{s}$ is the isothermal sound speed for $T \sim 10 \mathrm{~K}$ (André et al. 2010 - cf. Fig. 4-right). Note that the critical 

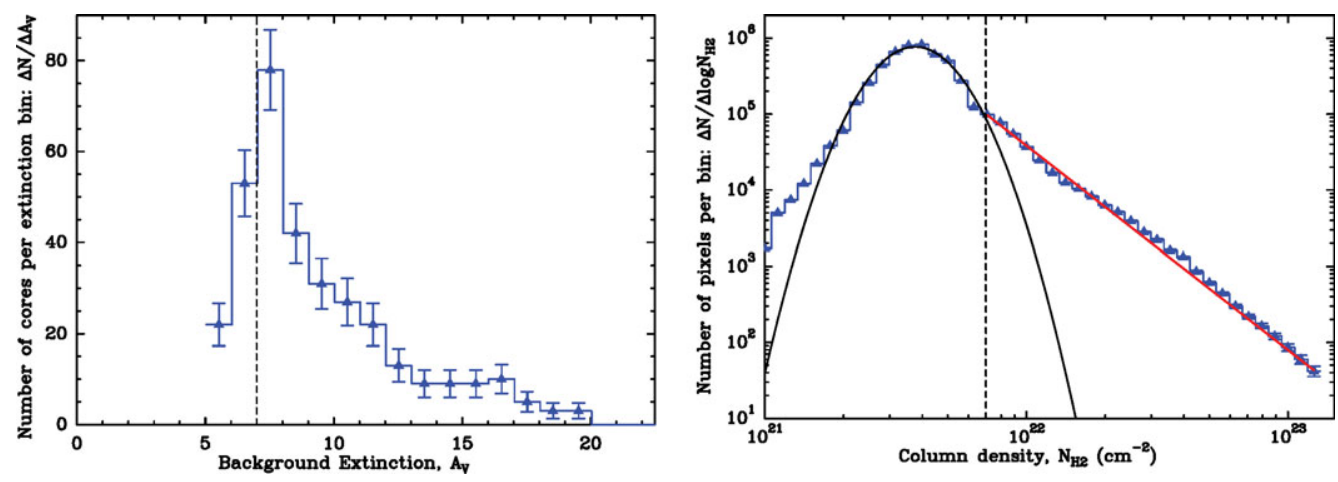

Figure 5. Left: Distribution of background column densities (converted to equivalent extinctions) for the best 341 candidate prestellar cores identified with Herschel in the Aquila Rift complex. Right: Probability density function of column density in the Aquila complex, based on the column density images derived from our Herschel data (cf. Könyves et al. 2010). A log-normal fit at low column densities and a power-law fit at high column densities are superimposed. In both panels, the vertical dashed line marks the extinction threshold $A_{V}^{\text {back }}=7$ (see $\S 3$ ).

line mass of a filament depends only on gas temperature and is modified by only a factor of order unity for filaments with realistic levels of magnetization (cf. Fiege \& Pudritz 2000). In contrast, in the non-star-forming, translucent Polaris cloud (where only unbound starless cores are found but no prestellar cores nor protostars), all of the filaments have subcritical masses per unit length (cf. Fig. 4-left), which is consistent with the view that they are gravitationally stable, hence neither collapsing nor forming stars.

The above scenario provides an explanation of the visual extinction threshold discussed in $\S 3$ above (cf. Fig. 5). Given the typical width $\sim 10000-15000$ AU measured for the filaments, the extinction threshold at $A_{V}^{\text {back }} \sim 7$ corresponds to within a factor of 2 to the critical mass per unit length $M_{\text {line,crit }} \sim 15 M_{\odot} /$ pc at $T \sim 10 \mathrm{~K}$. Thus, the extinction threshold approximately corresponds to the threshold above which the filaments are gravitationally unstable. Prestellar cores are only observed above this threshold because they form out of a filamentary background and only the supercritical, gravitationally unstable filaments are able to fragment into bound cores.

Interestingly, the median spacing between starless cores in Aquila is $\sim 16000 \mathrm{AU}$ which roughly matches the thermal Jeans length at $T \sim 10 \mathrm{~K}$ for a background column density corresponding to the $A_{V}^{\text {back }} \sim 7$ threshold. This is consistent with the idea that gravitational fragmentation is the dominant physical mechanism generating prestellar cores within the filaments. Naively, one would expect gravitational fragmentation to result in a narrow prestellar CMF sharply peaked at the median thermal Jeans mass. However, a broad CMF resembling the IMF (e.g. Fig. 3) can be produced if turbulence has generated a field of initial density fluctuations in the filaments (cf. Inutsuka 2001).

To conclude, our Herschel first results support the view that the form of the stellar IMF is largely inherited from the form of the prestellar CMF. They also reveal a tight connection between the large-scale $(>1 \mathrm{pc})$ filamentary structure of the parent clouds and the formation process of prestellar cores on small $(<0.1 \mathrm{pc})$ scales in the densest, gravitationally unstable parts of the filaments.

\section{References}

Alves, J. F., Lombardi, M., \& Lada, C. J. 2007, A\& A, 462, L17

André, Ph. , \& Saraceno, P. 2005, in The Dusty and Molecular Universe: A Prelude to Herschel and ALMA, ESA SP-577, p. 179 
André, P., Basu, S., \& Inutsuka, S.-I. 2009, in Structure Formation in Astrophysics, Ed. G. Chabrier, Cambridge University Press, p. 254

André, P., Belloche, A., Motte, F., \& Peretto, N. 2007, A\&A A, 472, 519

André, Ph., Men'shchikov, A., Bontemps, S. et al. 2010, A\&̇A, 518, L102

André, P., Ward-Thompson, D., Barsony, M. 2000, in Protostars and Planets IV, Eds. V. Mannings, A. P. Boss, \& S. S. Russell (Univ. of Arizona Press, Tucson), p. 59

Bate, M. R. \& Bonnell, I. A. 2005, MNRAS, 356, 1201

Bate, M. R., Bonnell, I. A., \& Bromm, V. 2003, MNRAS, 339, 577

Bontemps, S., André, Ph., Könyves, V. et al. 2010, A\& A, 518, L85

Chabrier, G. 2005, in The Initial Mass Function 50 years later, Eds. E. Corbelli et al., p.41

Elmegreen, B.G. \& Falgarone, E. 1996, ApJ, 471, 816

Enoch, M. L., Evans, N. J., Sargent, A. I. et al. 2008, ApJ, 684, 1240

Fiege, J. D., \& Pudritz, R. E. 2000, MNRAS, 311, 85

Goodwin, S. P., Nutter, D., Kroupa, P., Ward-Thompson, D., Whitworth, A. P. 2008, A\&\&A, 477,823

Guillout, P. 2001, in From Darkness to Light, Eds. T. Montmerle \& P. André, ASP Conf. Ser., 243 , p. 677

Gutermuth, R. A., Bourke, T. L., Allen, L. E. et al. 2008, ApJ, 673, L151

Heithausen, A. et al. 2002, ApJ, 383, 591

Hennebelle, P. \& Chabrier, G. 2008, ApJ, 684, 395

Inutsuka, S.-I. 2001, $A p J, 559$, L149

Inutsuka, S.-I. \& Miyama, S.M. 1997, ApJ, 480, 681

Johnstone, D., Wilson, C. D., Moriarty-Schieven, G., et al. 2000, ApJ, 545, 327

Johnstone, D., Fich, M., Mitchell, G. F., Moriarty-Schieven, G. 2001, ApJ, 559, 307

Johnstone, D., Di Francesco, J., \& Kirk, H. 2004, ApJ, 611, L45

Kainulainen, J., Beuther, H., Henning, T., \& Plume, R. 2009,A\&A, 508, L35

Klessen, R. S., \& Burkert, A. 2000, ApJS, 128, 287

Könyves, V., André, Ph., Men'shchikov, A. et al. 2010, A\& A, 518, L106

Kramer, C., Stutzki, J., Rohrig, R., Corneliussen, U. 1998, A\&A, 329, 249

Kroupa, P. 2001, MNRAS, 322, 231

Lada, C. J., Muench, A. A., Rathborne, J. M., Alves, J., \& Lombardi, M. 2008, ApJ, 672, 410

Larson, R. B., 1981, MNRAS, 194, 809

Larson, R. B. 1985, MNRAS, 214, 379

Men'shchikov, A., André, Ph., Didelon, P. et al. 2010, A\&3A, 518, L103

Miville-Deschênes, M.-A., Martin, P. G., Abergel, A. et al. 2010, A\&A, 518, L104

Molinari, S., Swinyard, B., Bally, J. et al. 2010, A\&A, 518, L100

Motte, F., André, P., Neri, R. 1998, A\&̛A, 336, 150

Motte, F., André, P., Ward-Thompson, D., \& Bontemps, S. 2001, AESA, 372, L41

Myers, P. C. 2009, ApJ, 700, 1609

Nutter, D. \& Ward-Thompson, D. 2007, MNRAS, 374, 1413

Onishi, T., Mizuno, A., Kawamura, A., Ogawa, H., Fukui, Y. 1998, ApJ, 502, 296

Onishi, T., Mizuno, A., Kawamura, A., Tachihara, K. \& Fukui, Y. 2002, ApJ, 575, 950

Ostriker, J. 1964, ApJ, 140, 1056

Padoan, P. \& Nordlund, A. 2002, ApJ, 576, 870

Padoan, P., Juvela, M., Goodman, A. A., \& Nordlund, A. 2001, ApJ, 553, 227

Pilbratt, G.L., Riedinger, J.R., Passvogel, T. et al. 2010, A\&A, 518, L1

Stamatellos, D., Whitworth, A. P., \& Ward-Thompson, D. 2007, MNRAS, 379, 1390

Stanke, T, Smith, M. D., Gredel, R., \& Khanzadyan, T. 2006, A\& $\&$, 447, 609

Starck, J. L., Donoho, D. L., Candès, E. J. 2003, A\&GA, 398, 785

Testi, L., Sargent, A. I. 1998, ApJ, 508, L91

Ward-Thompson, D., André, Ph., Crutcher, R., Johnstone, D., Onishi, T., \& Wilson, C. 2007, in Protostars $\mathscr{E}$ Planets $V$, Eds. B. Reipurth et al., Univ. of Arizona Press, p. 33

Ward-Thompson, D., Kirk, J.M., André, P. et al. 2010, A $\xi A$, 518, L92 